Pacific Journal of Mathematics

LOCAL AND GLOBAL BIFURCATION FROM NORMAL 


\title{
LOCAL AND GLOBAL BIFURCATION FROM NORMAL EIGENVALUES II
}

\author{
JoHN A. MACBAIN
}

\begin{abstract}
This paper studies the bifurcation of solutions of nonlinear eigenvalue problems of the form $L u=\lambda u+H(\lambda, u)$, where $L$ is linear and $H$ is $o(\|u\|)$ uniformly on bounded $\lambda$ intervals. This paper shows that isolated eigenvalues of $L$ having odd multiplicity are bifurcation points if $H$ merely has a "degree" of compactness, but is not necessarily compact (treated in [3], [5]). Moreover, a global alternative theorem follows.
\end{abstract}

Introduction. In this paper we study the bifurcation of solutions of nonlinear eigenvalue problems. The equations to be studied are of the form

$$
L u=\lambda u+H(u)
$$

where all operators are defined in a real Banach space $\mathscr{B} . \quad L$ is assumed to be linear, bounded or unbounded; $I$, the identity map, and $H, o(\|u\|)$ near $u=0$. Clearly, $(\lambda, 0)$ is a solution for each real $\lambda$, and these are called the trivial solutions of (0.1). Of more interest are the nontrivial solutions, pairs $(\lambda, u)$ satisfying (0.1) with $u \neq 0$. In particular, one is interested in how solutions of (0.1) are related to solutions of the linear equation

$$
L u=\lambda u \text {. }
$$

The study of this led to the following definition.

Definition. A point $\left(\lambda_{0}, 0\right)$ is a bifurcation point for $(0.1)$ if every neighborhood of $\left(\lambda_{0}, 0\right)$ in $\boldsymbol{R} \times \mathscr{B}$ contains a nontrivial solution of (0.1).

Under quite general conditions, it is easy to show that in order for $\left(\lambda_{0}, 0\right)$ to be a bifurcation point of $(0.1)$, it is necessary that $\lambda_{0}$ be in the spectrum of $L$. [8].

The first general existence theorem for bifurcation points was obtained by Krasnoseljskii [2]. He considered equations of the type

$$
u+\lambda L u+H(u)
$$

where $L$ is linear and compact, and $H$ compact. He proved that if $\lambda_{0}$ is a characteristic value of $L$ having odd algebraic multiplicity, 
then $\left(\lambda_{0}, 0\right)$ is a bifurcation point.

More recently, Rabinowitz [6] studied the same problem as Krasnoseljkii and proved a much stronger result. The bifurcation from such points is a global property, with a continuous branch of solutions joining $\left(\lambda_{0}, 0\right)$ to infinity in $R \times \mathscr{B}$, or if the branch is bounded, containing $\left(\lambda_{1}, 0\right)$ with $\lambda_{1} \neq \lambda_{0}$.

The author ([3] and [5]) eliminated the compactness assumption on $L$ while maintaining the strength of the result. The main result of this paper is that the compactness assumption on $H$ can be relaxed. The proofs of the theorems mentioned involve the use of degree theory.

1. Preliminaries. Let $\mathscr{B}$ be a real Banach space and let $\mathscr{E}$ denote $\boldsymbol{R} \times \mathscr{B}$ with the product topology. By a nonlinear eigenvalue problem we mean an equation of the form

$$
L u=\lambda u+H(u)
$$

where $L: \mathscr{B} \rightarrow \mathscr{B}$ is linear and $H: \mathscr{B} \rightarrow \mathscr{B}$ is a nonlinear operator satisfying hypothesis $H-1$ :

$(H-1)$ (i) $H$ is continuous, and bounded on each ball centered at 0 .

(ii) $H$ is $o(\|u\|)$ for $u$ near 0 .

A nontrivial solution of (1.1) is a pair $(\lambda, u)$ with $u \neq 0$ which satisfies (1.1), and the trivial solutions are the pairs $(\lambda, 0)$.

In what follows, $L: \mathscr{B} \rightarrow \mathscr{B}$ will be a densely defined linear operator (bounded or unbounded) with domain dom $(L)$. The resolvent set of $L, \rho(L)$, will be all real values of $\lambda$ for which there exists a bounded linear operator $C: \mathscr{B} \rightarrow \mathscr{B}$ such that

$$
\begin{aligned}
& C(L-\lambda) x=x, \quad x \in \operatorname{dom}(L) \\
& (L-\lambda) C x=x, \quad x \in \operatorname{range}(L-\lambda) .
\end{aligned}
$$

$C$ will be denoted by $(L-\lambda)^{-1}$.

Definition 1.1. The (algebraic) multiplicity of an eigenvalue $\lambda$ of $L$ is defined to be the dimension of the subspace $\bigcup_{j=1}^{\infty} \operatorname{ker}(L-\lambda)^{j}$ where $\operatorname{ker}(L-\lambda)^{j}$ denotes the nullspace of $(L-\lambda)^{j} . \bigcup_{j=1}^{\infty} \operatorname{ker}(L-\lambda)^{j}$ will be referred to as the principal manifold of $L$ associated with $\lambda$.

Definition 1.2. An eigenvalue $\lambda$ of $L$ is defined to be normal if

(i) the multiplicity of $L$ is finite.

(ii) $\mathscr{B}$ is the direct sum of subspaces $\mathscr{L}_{\lambda} \oplus \mathscr{N}_{\lambda}$ where $\mathscr{L}_{\lambda}=$ $\bigcup_{j=1}^{\infty} \operatorname{ker}(L-\lambda)^{j}, \mathscr{N}_{\lambda}$ is invariant under $L$, and $(L-\lambda)$ is invertible on $\mathscr{N}_{\lambda}$.

The projection of $\mathscr{B}$ onto $\mathscr{L}_{\lambda}$ along $\mathscr{N}_{\lambda}$ is denoted by $P_{\lambda}$. 
Hence $P_{\lambda} \mathscr{B}=\mathscr{L}_{\lambda}$ and $\left(I-P_{\lambda}\right) \mathscr{B}=\mathscr{N}_{\lambda}$. Let $Q_{\lambda}=I-P_{\lambda}$.

An eigenvalue $\lambda$ of $L$ is isolated if there exists $\varepsilon>0$ such that $(\lambda-\varepsilon, \lambda+\varepsilon)$ contains no other members of $\mathrm{sp} L$. The set of isolated normal eigenvalues of $L$ is called the discrete spectrum of $L$ which we denote by $\operatorname{sp}_{d}(L)$. The remaining part of the spectrum will be called nondiscrete and is denoted by $\mathrm{sp}_{n d}(L)$.

REMARK. If $L$ is self-adjoint, the nondiscrete spectrum is the essential spectrum of $L$.

DEFINITION 1.3. $(\lambda, 0)$ is a bifurcation point for (1.1) if every neighborhood in $\mathscr{E}$ of $(\lambda, 0)$ contains a nontrivial solution of (1.1).

Definition 1.4. If $\mathscr{V}$ is a subset of $\mathscr{E}, \mathscr{\mathscr { S }}$, and $\mathscr{V}_{R}$ are defined to be $\mathscr{V}^{\lambda}=\{u \mid(\lambda, u) \in \mathscr{V}\}$ and $\mathscr{V}_{\boldsymbol{R}}=\{\lambda \mid(\lambda, u) \in \mathscr{V}$ for some $u\}$. For $W \subset \boldsymbol{R}, \mathscr{B}$, or $\mathscr{E}, \bar{W}$ denotes the closure of $W$ in the respective space.

Some of the material that follows in this section was presented in [8], and is repeated here without proof.

Definition. The set measure of compactness of a bounded set $\Omega$, expressed by $\alpha(\Omega)$, is defined to be the infimum of all $\delta>0 \mathrm{such}$ that $\Omega$ can be covered by a finite number of balls having radius $\delta$.

Some useful results in this area include:

(i ) $\alpha(\Omega)=\alpha(\bar{\Omega})$ for all bounded sets $\Omega$.

(ii) If $\Omega$ is bounded, $\Omega$ is relatively compact if and only if $\alpha(\Omega)=0$.

(iii) $\alpha\left(\Omega_{1}+\Omega_{2}\right) \leqq \alpha\left(\Omega_{1}\right)+\alpha\left(\Omega_{2}\right)$.

(iv) If $\lim _{n \rightarrow \infty} x_{n}=0$, then $\alpha\left(\left\{x_{n}\right\}_{n=1,2}, \ldots\right)=0$.

Definition. An operator $T: B \rightarrow B$ is called a $k$-set contraction if it is continuous and $\alpha(T(\Omega)) \leqq k \alpha(\Omega)$ for all bounded sets $\Omega$. Let $\gamma(T)=\inf \{k \mid T$ is a $k$-set contraction $\}$. The following results concerning $k$-set contractions hold.

(i) $T$ is compact if and only if $T$ is a 0 -set contraction.

(ii) If $L$ is a bounded linear operator with operator norm $\|L\|$, then $L$ is a $\|L\|$-set contraction. (This need not be true if $L$ is nonlinear. (See $\S 4$.))

(iii) If $L$ is a bounded, linear, and self-adjoint operator, $\gamma(L)=$ $\rho_{e}(L)$ where $\rho_{e}(L)$ is the radius of the essential spectrum of $L$. [8].

(iv) If $F=G H$ with $G$ linear, $\gamma(F) \leqq\|G\| \gamma(H)$. In general, for all $G$ and $H, \gamma(F) \leqq \gamma(G) \gamma(H)$.

A degree theory for nonlinear operators of the form $I-T$, where $T: B \rightarrow B$ is a $k$-set contraction with $k<1$, was developed by 
Nussbaum in his thesis. The results of Nussbaum's to be used are given below, together with a theorem of Stuart.

Let $T: B \rightarrow B$ be a $k$-set contraction $(k<1)$. Then an integervalued function, denoted by deg, can be defined so as to have the following properties.

(1) $\operatorname{deg}(\Omega, I-T, 0)$ is well defined for each open, bounded subset $\Omega \subset B$ such that $T$ has no fixed points on the boundary $\partial \Omega$ of $\Omega$.

(2) If $\operatorname{deg}(\Omega, I-T, 0) \neq 0$, then there is a point $x \in \Omega$ such that $x=T x$.

(3) If $\Omega_{1}$ and $\Omega_{2}$ are open subsets of $\Omega$, itself a bounded, open subset of such that $T$ has no fixed points in $\left.\left[\bar{\Omega} \mid\left(\Omega_{1} \cup \Omega_{2}\right)\right] \cup \overline{\left(\Omega_{1} \cap \Omega_{2}\right.}\right)$, then $\operatorname{deg}(\Omega, I-T, 0)=\operatorname{deg}\left(\Omega_{1}, I-T, 0\right)+\operatorname{deg}\left(\Omega_{2}, I-T, 0\right)$.

(4) If $T$ is compact, then $\operatorname{deg}(\Omega, I-T, 0)=d(\Omega, I-T, 0)$, where $d$ denotes the Leray-Schauder degree, whenever the left-hand side is defined. [8].

The arguments of this paper will closely follow those of [5]. Thus, a notation of index is helpful. Define

$$
\text { index }\left(T, x_{0}\right)=\operatorname{deg}(B, I-T, 0)
$$

where $B$ is an open ball in $B$ centered at $x_{0}$ with a radius small enough so that $x_{0}$ is the only fixed point of $T$ in $\bar{B}$.

In [5], critical use was made of a theorem in Leray-Schauder degree theory which has been extended to the Nussbaum degree theory by Toland and Stuart [8].

THEOREM. Let $T: X \rightarrow X$ be a k-set contraction $(k<1)$ and let $x_{0}$ be a fixed point of $T$. Suppose that $T$ has Frechet derivative $T^{\prime \prime}\left(x_{0}\right)$ at $x_{0}$ and that unity is not an eigenvalue of $T^{\prime}\left(x_{0}\right)$.

Then $x_{0}$ is an isolated fixed point of $T$, and

$$
\text { ind }\left(T, x_{0}\right)=(-1)^{\nu} \text {, }
$$

where $\nu$ is the sum of the multiplicities of the eigenvalues greater than unity of $T^{\prime}\left(x_{0}\right)$.

Proof. See [8].

2. Local bifurcation theorem. The first theorem shows that bifurcation from an isolated eigenvalue $\lambda_{0}$ of $L$ having odd multiplicity is not dependent upon $H$ being compact, but rather on how "close" $H$ is to being compact.

THeOREM 2.1. Let $L$ be as above and let $H$ satisfy $H-1 . \quad \lambda_{0}$ 
is an isolated normal eigenvalue of $L$ having odd multiplicity. Assume that for $\left|\lambda-\lambda_{0}\right|<\varepsilon^{\prime}$, $\left\|(L-\lambda)^{-1} Q_{\lambda_{0}}\right\| \gamma(H) \leqq K<1$. Then, $\left(\lambda_{0}, 0\right)$ is a bifurcation point for (1.1).

Proof. In order to prove this theorem, (1.1) will be rewritten in the form $u-C(\lambda, u)=0$. Split (1.1) by

$$
\begin{aligned}
& L P_{\lambda_{0}} u=\lambda P_{\lambda_{0}} u+P_{\lambda_{0}} H(\lambda, u) \\
& L Q_{\lambda_{0}} u=\lambda Q_{\lambda_{0}} u+Q_{\lambda_{0}} H(\lambda, u) .
\end{aligned}
$$

A solution of (1.1) is equivalent to a simulation solution of the two equations in (2.1). Select $\mu_{0} \in \rho(L)$. Instead of (2.1) we may write

$$
\begin{aligned}
& P_{\lambda_{0}} u=\frac{\left(L-\mu_{0}\right) P_{\lambda_{0}} u}{\lambda-\mu_{0}}-\frac{P_{\lambda_{0}} H(\lambda, u)}{\lambda-{ }_{0} \mu} \\
& Q_{\lambda_{0}} u=(L-\lambda)^{-1} Q_{\lambda_{0}} H(\lambda, u)
\end{aligned}
$$

where $(L-\lambda)^{-1}$ is to be interpreted as $(L-\lambda)^{-1} \mid \mathscr{N}_{\lambda_{0}}$. Thus, (2.2) is valid for $\lambda \in\left\{\lambda_{0}\right\} \cup\left\{\rho(L) \backslash\left\{\mu_{0}\right\}\right\}$. Adding these equations we get

$$
\begin{aligned}
u & =C_{1}(\lambda, u)+C_{2}(\lambda, u) \\
C_{1}(\lambda, u) & =\frac{\left(L-\mu_{0}\right) P_{\lambda_{0}} u}{\lambda-\mu_{0}} \\
C_{2}(\lambda, u) & =\left((L-\lambda)^{-1} Q_{\lambda_{0}}-\frac{P_{\lambda_{0}}}{\lambda-\mu_{0}}\right) H .
\end{aligned}
$$

Note that $C_{1}: \mathscr{E} \rightarrow \mathscr{B}$ is compact and linear in $u$ for each fixed $\lambda$ $C_{2}: \mathscr{E} \rightarrow \mathscr{B}$ satisfies $H-1$. Define

$$
\Phi(\lambda, \cdot)=I-C_{1}(\lambda, \cdot)-C_{2}(\lambda, \cdot) .
$$

Clearly, (2.3) or $\Phi(\lambda, u)=0$ is equivalent to (1.1) for the specified values of $\lambda$ when $L$ is bounded. If $L$ is unbounded, the question arises as to wheter $u$ is in $\operatorname{dom}(L)$ if $(\lambda, u)$ is a zero of $\Phi$. Noting (2.2), which is obtained from (2.3) by projecting onto $\mathscr{L}_{\lambda_{0}}, \mathscr{N}_{\lambda_{0}}$ respectively, we see that $Q_{\lambda_{0}} u$ is in $\operatorname{dom}(L)$. Since $P_{\lambda_{0}} u$ is in an eigenspace of $L, u=P_{\lambda_{0}} u+Q_{\lambda_{0}} u$ is in $\operatorname{dom}(L)$.

If the assertion of the theorem is not true we can find a neighborhood $\mathcal{O}$ of $\left(\lambda_{0}, 0\right)$ such that the only solutions of (1.1) in $\mathcal{O}$ are trivial solutions, $\rho(L) \backslash \mathscr{O}_{\boldsymbol{R}} \neq \varnothing$, and $\mathscr{O}_{\boldsymbol{R}} \cap \operatorname{sp} L=\left\{\lambda_{0}\right\}$. Select $\mu_{0} \in$ $\rho(L) \backslash \mathcal{O}_{R}$ such that (1.1) is equivalent to (2.3) for all $\mathscr{O}_{\boldsymbol{R}}$. Select $\varepsilon>0, \quad 0<\varepsilon<\varepsilon^{\prime}$, that $\left[-\varepsilon+\lambda_{0}, \lambda_{0}+\varepsilon\right] \times\{0\} \subset \mathcal{O}$. Applying the homotopy property of degree theory we obtain

$$
\operatorname{deg}\left(\Phi(\lambda, \cdot), O^{\lambda}, 0\right)=\text { constant, }\left|\lambda-\lambda_{0}\right|<\varepsilon .
$$

Select $\underline{\lambda}$ and $\bar{\lambda}$ such that $\lambda_{0}-\varepsilon<\underline{\lambda}<\lambda_{0}<\bar{\lambda}<\lambda_{0}+\varepsilon$. Then 


$$
\begin{aligned}
& \operatorname{deg}\left(\Phi(\lambda, \cdot), \mathcal{O}^{\underline{\lambda}}, 0\right)=\operatorname{index}\left(I-C_{1}(\underline{\lambda}, \cdot),(\underline{\lambda}, 0)\right) \\
& \operatorname{deg}\left(\Phi(\bar{\lambda}, \cdot), \mathcal{O}^{\bar{\lambda}}, 0\right)=\operatorname{index}\left(I-C_{1}(\bar{\lambda}, \cdot),(\bar{\lambda}, 0)\right) .
\end{aligned}
$$

Thus, using (2.5) and (2.6),

$$
\begin{aligned}
& \text { index }\left(I-C_{1}(\underline{\lambda}, \cdot),(\underline{\lambda}, 0)\right) \\
& \quad=\operatorname{index}\left(I-C_{1}(\bar{\lambda}, \cdot \cdot),(\bar{\lambda}, 0)\right) .
\end{aligned}
$$

However, since the multiplicity of $\lambda_{0}$ is odd,

$$
\begin{aligned}
& \text { index }\left(I-C_{1}(\underline{\lambda}, \cdot),(\underline{\lambda}, 0)\right) \\
& \quad=-\operatorname{index}\left(I-C_{1}(\bar{\lambda}, \cdot \cdot),(\bar{\lambda}, 0)\right) .
\end{aligned}
$$

Since the indices in (2.7) and (2.8) are either +1 or -1 , we have a contradiction. Thus, such a neighborhood can never be found. This proves that $\left(\lambda_{0}, 0\right)$ is a bifurcation point.

REMARK 1. If $\lambda_{0} \neq 0$ is an eigenvalue of $L$ having odd multiplicity, then the hypotheses of Theorem 1 are satisfied if $L$ is compact or if $L$ is self-adjoint with $\lambda_{0}$ isolated in $\operatorname{sp} L$.

REMARK 2. The condition on $\gamma\left((L-\lambda)^{-1} Q_{\lambda_{0}} H\right)$ can be relaxed. If one restricted the operators to a ball $B_{0}$ centered at $\left(\lambda_{0}, 0\right)$ and then extended them to all of $R \times B_{0}$ in a linear manner, one could apply Theorem 1.1 if $\gamma\left((L-\lambda)^{-1} Q_{\lambda_{0}} H \mid B_{0}\right)=K<1$, for $\left|\lambda-\lambda_{0}\right|<\varepsilon^{\prime}$. This would handle the case that $H$ is well behaved near $u=0$ but grows too large for $u$ far from 0 .

$(H-2) \quad H: \mathscr{E} \rightarrow \mathscr{B}$ satisfies:

(i) $H$ is continuous, and bounded on each ball centered at 0 .

(ii) $H$ is $o(\|u\|)$ uniformly on bounded $\lambda$ intervals.

REMARK 3. The theorem remains true if $H$ satisfies hypothesis $H-2$ rather than the more restricted $H-1$. The proof is very similar.

3. A global alternative theorem. In this section we will show that the local bifurcation exhibited in Theorem 2.1 is a global property with an alternative-type result.

For $\mathscr{V} \subset \mathscr{E}$, a subcontinuum of $\mathscr{V}$ is a subset of $\mathscr{V}$ which is closed and connected in $\mathscr{E}$. $\mathscr{S}$ will denote the closure of the set of nontrivial solutions of (1.1) in $\mathscr{E}$. Let $\mathscr{C}_{\lambda_{0}}$ denote the maximal subcontinuum of $\mathscr{S} \cup\left(\lambda_{0}, 0\right)$ containing $\left(\lambda_{0}, 0\right) . \quad B_{\rho}$ will denote the open ball in $\mathscr{B}$ centered at 0 and having radius $\rho . L$ and $H$ will be as in $\S 2$. 
LEMma 3.1. Suppose $\lambda_{0}$ and $\lambda_{1}$ are distinct normal eigenvalues of $L$. Then $\mathscr{B}=\mathscr{L}_{\lambda_{0}} \oplus \mathscr{L}_{\lambda_{1}} \oplus \mathscr{N}$, a direct sum of subspaces, where $\mathscr{N}=\mathscr{N}_{\lambda_{0}} \cap \mathscr{N}_{\lambda_{1}}$, and $P=P_{\lambda_{0}}+P_{\lambda_{1}}$ projects onto $\mathscr{L}_{\lambda_{0}} \oplus \mathscr{L}_{\lambda_{1}}$ along $\mathscr{N} \cdot[5]$.

Lemma 3.2. Let $K$ be a compact metric space and $A$ and $B$ disjoint closed subsets of $K$. Then either there exists a subcontinuum of $K$ meeting both $A$ and $B$, or $K=K_{A} \cup K_{B}$ where $K_{A}$ and $K_{B}$ are disjoint compact subsets of $K$ containing $A$ and $B$ respectively.

Proof. See [9].

For $\lambda_{0}$ as before, define

$$
\begin{aligned}
& \alpha_{1}\left(\lambda_{0}\right)=\sup \left\{\lambda \mid \lambda<\lambda_{0}, \lambda \in \operatorname{sp}_{n d}(L)\right\} \\
& \beta_{1}\left(\lambda_{0}\right)=\inf \left\{\lambda \mid \lambda>\lambda_{0}, \lambda \in \operatorname{sp}_{n d}(L)\right\} .
\end{aligned}
$$

These values will be $\pm \infty$ respectively if the vacuous case results. For $\varepsilon_{j}>0$, consider $I\left(\varepsilon_{1}, \varepsilon_{2}\right)=\left[\alpha_{1}\left(\lambda_{0}\right)+\varepsilon_{1}, \beta_{1}\left(\lambda_{0}\right)-\varepsilon_{2}\right]$. (Here assume both are finite.) Let $P_{\varepsilon_{1}, \varepsilon_{2}}=\sum P_{\lambda}$ where the summation is over all eigenvalues of $L$ in $I\left(\varepsilon_{1}, \varepsilon_{2}\right)$, and let $Q_{\varepsilon_{1}, \varepsilon_{2}}=I-P_{\varepsilon_{1}, \varepsilon_{2}}$. Select $\varepsilon_{1}>0$ and $\varepsilon_{2}>0$ such that $\left\|(L-\lambda)^{-1} Q_{\varepsilon_{1}, \varepsilon_{2}}\right\| \gamma(H)<1$ on $I\left(\varepsilon_{1}, \varepsilon_{2}\right)$. Let $\left[\alpha\left(\lambda_{0}\right), \beta\left(\lambda_{0}\right)\right]=I\left(\varepsilon_{1}, \varepsilon_{2}\right)$. If $\alpha_{1}\left(\lambda_{0}\right)$ or $\beta_{1}\left(\lambda_{0}\right)$ are infinite, select $\alpha\left(\lambda_{0}\right)$ or $\beta\left(\lambda_{0}\right)$ to be any appropriate finite number.

LEMma 3.3 Suppose $\lambda_{0}$ is an isolated normal eigenvalue of $L$ having finite multiplicity. Assume $\mathscr{C}_{\lambda_{0}}$ is bounded, $\left(\overline{\mathscr{C}_{\lambda_{0}}}\right)_{\boldsymbol{R}} \cap$ $\left\{\alpha\left(\lambda_{0}\right), \beta\left(\lambda_{0}\right)\right\}=\phi$, and $\mathscr{C}_{\lambda_{0}} \cap\{\boldsymbol{R} \times\{0\}\}=\left(\lambda_{0}, 0\right)$. Then $\mathscr{C}_{\lambda_{0}}$ is compact and there exists a bounded open set $\mathcal{O}$ such that $\mathscr{C}_{\lambda_{0}} \subset \mathcal{O}$, $\partial \mathcal{O} \cap$ $\mathscr{S}=\varnothing,\left(\overline{\mathscr{O}_{R}}\right) \cap \mathrm{sp}_{n d}(L)=\varnothing$, the trivial solutions contained in $\varnothing$ are the points $(\lambda, 0)$ where $\left|\lambda-\lambda_{0}\right|<\varepsilon$ for some $\varepsilon<\varepsilon_{0}=\operatorname{dist}\left(\lambda_{0}\right.$, $\left.\operatorname{sp} L \backslash\left\{\lambda_{0}\right\}\right)$, and $\|(\lambda, u)-(\mu, 0)\| \geqq 2 \varepsilon_{1}$ for some positive $\varepsilon_{1}$ whenever $(\lambda, u) \in \partial \mathcal{O}$ and $\mu \varepsilon \operatorname{sp} L$.

Proof. $\mathscr{C}_{\lambda_{0}}$ is compact. Indeed, let $\left\{\left(\lambda_{n}, u_{n}\right)\right\}_{n \in \mathscr{N}}$ be elements of $\mathscr{C}_{\lambda_{0}}$. Since $\mathscr{C}_{\lambda_{0}}$ is bounded, we may find a $\lambda$ and a subsequence $\mathscr{N}_{1}$ such that $\lim _{\substack{n \rightarrow \infty \\ n \in \mathcal{C}_{1}}} \lambda_{n}=\lambda$. Let $P$ be the projection for $\left(\overline{\mathscr{C}_{\lambda_{0}}}\right)_{R}$ and $Q=I-P$. Consider $\left\{u_{n}\right\}_{n \in \mathscr{N}_{1}}$.

$$
\begin{aligned}
\alpha\left(\left\{u_{n}\right\}_{n \in \mathscr{N}_{1}}\right) & =\alpha\left(\left\{C_{1}\left(\lambda_{n}, u_{n}\right)+C_{2}\left(\lambda_{n}, u_{n}\right)\right\}_{n \in \mathscr{N}_{1}}\right) \\
& \leqq \alpha\left(\left\{C_{1}\left(\lambda_{n}, u_{n}\right)\right\}_{n \in \mathscr{N}_{1}}+\left\{C_{2}\left(\lambda_{n}, u_{n}\right)\right\}_{n \in \mathscr{S}_{1}}\right) \\
& \leqq \alpha\left(\left\{C_{2}\left(\lambda_{n}, u_{n}\right)\right\}_{n \in \mathscr{N}_{1}}\right)
\end{aligned}
$$




$$
\begin{aligned}
\text { (similarly) } \leqq & \alpha\left(\left\{\left(L-\lambda_{n}\right)^{-1} Q H\left(u_{n}\right)\right\}_{n \in \mathscr{r}_{1}}\right) \\
\leqq & \alpha\left(\left\{(L-\lambda)^{-1} Q H\left(u_{n}\right)\right\}_{n \in \mathscr{r}_{1}}\right) \\
& +\alpha\left(\left\{\left(\left(L-\lambda_{n}\right)^{-1}-(L-\lambda)^{-1}\right) Q H\left(u_{n}\right)\right\}_{n \in \mathscr{S}_{1}}\right) \\
= & \alpha\left(\left\{(L-\lambda)^{-1} Q H\left(u_{n}\right)\right\}_{n \in \mathscr{r}_{1}}\right) \\
\leqq & \left\|(L-\lambda)^{-1} Q\right\| \alpha\left(\left\{H\left(u_{n}\right)\right\}_{n \in \mathscr{N}_{1}}\right) \\
\leqq & \left\|(L-\lambda)^{-1} Q\right\| \gamma(H) \alpha\left(\left\{u_{n}\right\}_{n \in \mathscr{N}_{1}}\right) \\
< & \alpha\left(\left\{u_{n}\right\}_{n \in \mathscr{N}_{1}}\right) .
\end{aligned}
$$

Thus $\alpha\left(\left\{u_{n}\right\}_{n \in \mathscr{N}_{1}}\right)=0$ meaning the set is compact, meaning it has a convergent subsequence. Thus, $C_{\lambda_{0}}$ is compact.

The remainder of the proof follows from [5] and [8] using Lemma 3.1.

The following theorem is modeled after one in [5] given for the case when $H$ is compact.

THEOREM 3.1. Suppose $\lambda_{0}$ is an isolated eigenvalue of $L$ of odd multiplicity. $L$ is as before and $H$ satisfies $H-1$. Furthermore, let $\left\|\left(L-\lambda_{0}\right)^{-1} Q_{\lambda_{0}}\right\| \gamma(H)<1$. Then $\left(\lambda_{0}, 0\right)$ is a bifurcation point of (1.1) possessing a maximal continuous branch $\mathscr{C}_{\lambda_{0}}$ such that exactly one of the following alternatives occurs.

(i) $\mathscr{C}_{\lambda_{0}}$ is unbounded.

(ii) $\mathscr{C}_{\lambda_{0}}$ is bounded and $\left(\overline{\mathscr{C}_{\lambda_{0}}}\right)_{R} \cap\left\{\alpha\left(\lambda_{0}\right), \beta\left(\lambda_{0}\right)\right\} \neq 0$.

(iii) $\mathscr{C}_{\lambda_{0}}$ is compact, $\left(\mathscr{C}_{\lambda_{0}}\right)_{\boldsymbol{R}} \cap\left\{\alpha\left(\lambda_{0}\right), \beta\left(\lambda_{0}\right)\right\}=\varnothing$ and $\mathscr{C}_{\lambda_{0}} \cap\{\boldsymbol{R} \times\{0\}\}=$ $\left\{\lambda_{0}, \lambda_{1}, \cdots, \lambda_{n}\right\} \times\{0\}$ where $\lambda_{1}, \cdots, \lambda_{n}$ are normal eigenvalues of $L$ distinct from $\lambda_{0}$, and the sum of the multiplicities of $\lambda_{0}, \lambda_{1}, \cdots, \lambda_{n}$ is even.

Proof. With the use of Lemma 3.3, the proof is similar to Theorem 2.1 and Theorem 2.2 [5].

REMARK 1. The hypotheses of this theorem are unnecessarily stringent. The same results hold with $H(\lambda, u)$ if $\left\|\left(L-\lambda_{0}\right)^{-1} Q_{\lambda_{0}}\right\| \gamma\left(H\left(\lambda_{0}, \cdot\right)\right)<$ 1, where $H$ satisfies $H-2$. The preceding proofs, however, become a little more complicated mainly due to notation.

REMARK 2. Suppose that $H(\lambda, \cdot)$, when restricted to a ball centered at $u=0$, has $\left\|\left(L-\lambda_{0}\right)^{-1} Q_{\lambda_{0}}\right\| \gamma\left(H_{\left(\lambda_{0}, .\right)}\right)<1$ (but this hypothesis fails on the entire space). One can do the degree work on these balls (by reworking all previous proofs) and obtain a theorem similar to that in [4]. (It was necessary to make a change in that theorem due to an error committed in [4] (see the next section).)

Assign $F(\varepsilon)=\left[\alpha\left(\lambda_{0}\right)+\varepsilon, \beta\left(\lambda_{0}\right)-\varepsilon\right]$. Let $P_{\varepsilon}$ correspond to $F(\varepsilon)$, and $Q_{\varepsilon}=I-P_{\varepsilon}$. When restricted to a ball of radius $r$ centered at 
0 , let $H_{(\lambda, \cdot)}$ be a $\gamma_{r}(H(\lambda, \cdot))$-set contraction, and define $\gamma_{r}(H)$ to be strictly monotone increasing.

THEOREM 3.2. Let $\lambda_{0}$ be an isolated eigenvalue of $L$ having odd algebraic multiplicity. $L$ is as before and $H$ satisfies $H-2$. Then $\left(\lambda_{0}, 0\right)$ is a bifurcation point of (1.1) and emanating from it is a maximal continuous branch $\mathscr{C}_{\lambda_{0}}$ which obeys exactly one of the following alternatives for each suitably small $\varepsilon>0$.

(i) $\mathscr{C}_{\lambda_{0}}$ is unbounded.

(ii) $\mathscr{C}_{\lambda_{0}}$ is bounded and $\overline{\mathscr{C}_{\lambda_{0}}}$ meets $S_{\varepsilon}=\{(\lambda, u) \mid \lambda \in F(\varepsilon)$ and $\|u\|=r$ where $\left.\gamma_{r}(H(\lambda, \cdot))=\left\|(L-\lambda)^{-1} Q_{\varepsilon}\right\|^{-1}\right\} \cup\left\{\left(\alpha\left(\lambda_{0}\right)+\varepsilon\right) \times \mathscr{B}\right\} \cup$ $\left\{\left(\beta\left(\lambda_{0}\right)-\varepsilon\right) \times \mathscr{B}\right.$.

(iii) $\mathscr{C}_{\lambda_{0}}$ is compact, $\overline{\mathscr{C}_{\lambda_{0}}}$ does not meet $S_{\varepsilon}$, and $\mathscr{C}_{\lambda_{0}} \cap\{0 \times B\}=$ $\left\{\lambda_{0}, \lambda_{1}, \cdots, \lambda_{n}\right\}$, each a distinct normal eigenvalue of $L$, and the sum of their algebraic multiplicities is even.

REMARK 1. In the case where $L$ is self-adjoint, $\left\|(L-\lambda)^{-1} Q_{\varepsilon}\right\|=$ $1 /$ dist $(\lambda, \operatorname{sp}(L) / F(\varepsilon))$ where dist $(\cdot)$ is the standard distance function in $\boldsymbol{R}$. This simplifies the statement of (ii).

REMARK 2. If $\left(\alpha\left(\lambda_{0}\right), \beta\left(\lambda_{0}\right)\right) \cap \mathrm{sp}(L)$ consists of a finite list of eigenvalues, there is an $\varepsilon_{0}>0$ such that whenever $0<\varepsilon_{1}<\varepsilon_{2} \leqq \varepsilon_{0}$, $S_{\varepsilon_{1}}$ and $S_{\varepsilon_{2}}$ are identical in $F\left(\varepsilon_{2}\right) \times B$. This is because $\left\|(L-\lambda)^{-1} Q_{\varepsilon}\right\|$ is constant in $\varepsilon$ for $0<\varepsilon<\varepsilon_{0}$. This leads to an improvement in (ii) and (iii).

(ii $)^{\prime} \mathscr{C}_{\lambda_{0}}$ is bounded and $\overline{\mathscr{C}_{\lambda_{0}}}$ meets $S=\left\{(\lambda, u) \mid \lambda \in\left(\alpha\left(\lambda_{0}\right), \beta\left(\lambda_{0}\right)\right)\right.$ and $\|u\|=r$ where $\left.\gamma_{r}(H(\lambda, \cdot))=\left\|(L-\lambda)^{-1} Q_{\varepsilon_{0}}\right\|^{-1}\right\} \cup\left\{\alpha\left(\lambda_{0}\right) \times B\right\} \cup$ $\left\{\beta\left(\lambda_{0}\right) \times B\right\}$.

(iii) $\mathscr{C}_{\lambda_{0}}$ is compact, $\overline{\mathscr{C}_{\lambda_{0}}}$ does not meet $S$, and $\mathscr{C}_{\lambda_{0}} \cap\{0 \times B\}=$ $\left\{\lambda_{0}, \lambda_{1}, \cdots, \lambda_{n}\right\}$, each a distinct normal eigenvalue of $L$, and the sum of their algebraic multiplicities is even.

4. Other results. The theorems I proposed in [4] are unfortunately incorrect as stated and require modification as in $\S 3$ of this paper. The hypothesis of continuity on $H$ had to be strengthened. My error was in a proof that if one restricted $H$ to a ball centered at 0 in $B$ and on that ball $\|H\|=k$, then $H$ was a $k$-set contraction on the ball. This is true for linear operators.

This error was found by Professor Norman Dancer, The University of New England, Armidale N.S.W., Australia. He constructed a counterexample to Theorem I of [4], which I present here. There is an operator $V: c_{0} \rightarrow c_{0}$ such that if $x=\lambda V(x)$, then $x=0$ and $\lambda=0$. Set $B=c_{0} \times R, L: B \rightarrow B$ is defined by $L(w, t)=$ $(2 w, t)$ and $H: R \times B \rightarrow B$ is defined by $H\left(\lambda,(w, t)=\left(0, \lambda t^{2} V(w)\right)\right.$. 
$\lambda=1$ is an eigenvalue of $L$ of multiplicity 1 . If $\lambda$ is near 1 and $L u=\lambda u+H(\lambda, u)$ where $u=(w, t)$, then $t=\lambda t$ and $2 w=\lambda w+$ $\lambda t^{2} V(w) . \quad w=\lambda t^{2} V(w) /(2-\lambda)$ which implies $w=0$, and together with $\lambda$ being near 1 imply $t=0$. Thus, for $\lambda$ near 1 , the only solution is $u=0$. Many thanks to Professor Dancer. The operator $V$ is due to Ana and Vasile Istratescu and appeared in the Proceeding of the Amer. Math. Soc., Vol. 48, No. 1, page 197.

\section{REFERENCES}

1. N. Dunford and J. T. Schwarz, Linear Operators, Part II, Interscience, New York, 1963).

2. M. A. Krasnoseljskii, Topological Methods in the Theory of Nonlinear Integral Equations, Pergamon Press, New York, 1964.

3. J. A. MacBain, Global bifurcation theorems for noncompact operators, Bulletin Amer. Math. Soc., 80 (1974),

4. - Global bifurcation theorems for nonlinearly perturbed operator equation, Bulletin Amer. Math. Soc., 82 (1976).

5. — Local and global bifurcation from normal eigenvalues, Pacific J. Math., 63 (1976), 445-466.

6. P. H. Rabinowitz, Some aspects of nonlinear eigenvalue problems, Rocky Mountain

J. Math., 3 (1973).

7. F. Riesz, B. Sz.-Nagy, Functional Analysis, tr. L. Boron, Ungar, New York, 1971.

8. C. A. Stuart, Some bifurcation theory for $k$-set contractions, Proc. London Math. Soc., (1973).

9. G. T. Whyburn, Topological Analysis, Princeton University Press, Princeton, 1958.

Received February 16, 1977.

Air Force Institute of Technology

Wright-Patterson AIr ForCe Base, OH 45433 


\section{PACIFIC JOURNAL OF MATHEMATICS}

\section{EDITORS}

RICHARD ARENS (Managing Editor)

University of California

Los Angeles, California 90024

C. W. Curtis

University of Oregon

Eugene, OR 97403

C. C. MOORE

University of California

Berkeley, CA 94720

\section{J. DUGUNDJI}

Department of Mathematics University of Southern California Los Angeles, California 90007

R. Finn AND J. Milgram Stanford University Stanford, California 94305

ASSOCIATE EDITORS
E. F. BECKENBACH

B. H. NeUMANN

F. WOLF

K. YoshidA

\section{SUPPORTING INSTITUTIONS}

UNIVERSITY OF BRITISH COLUMBIA UNIVERSITY OF SOUTHERN CALIFORNIA CALIFORNIA INSTITUTE OF TECHNOLOGY STANFORD UNIVERSITY UNIVERSITY OF CALIFORNIA MONTANA STATE UNIVERSITY UNIVERSITY OF TOKYO UNIVERSITY OF NEVADA, RENO UNIVERSITY OF UTAH NEW MEXICO STATE UNIVERSITY OREGON STATE UNIVERSITY UNIVERSITY OF OREGON

WASHINGTON STATE UNIVERSITY UNIVERSITY OF WASHINGTON OSAKA UNIVERSITY 


\section{Pacific Journal of Mathematics}

\section{Vol. 74, No. $1 \quad$ May, 1978}

Gerald Arthur Anderson, Computation of the surgery obstruction groups

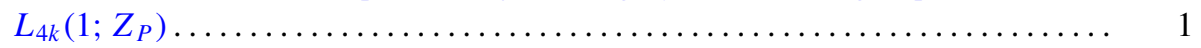

R. K. Beatson, The degree of monotone approximation ................ 5

Sterling K. Berberian, The character space of the algebra of regulated functions . . . 15

Douglas Michael Campbell and Jack Wayne Lamoreaux, Continua in the plane with

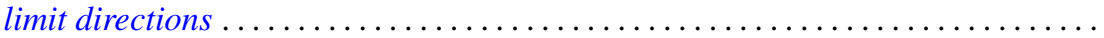

R. J. Duffin, Algorithms for localizing roots of a polynomial and the Pisot

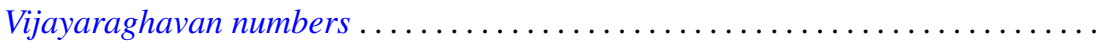

Alessandro Figà-Talamanca and Massimo A. Picardello, Functions that operate on

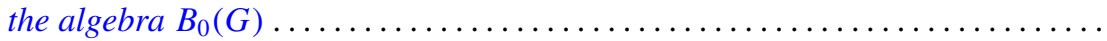

John Erik Fornaess, Biholomorphic mappings between weakly pseudoconvex

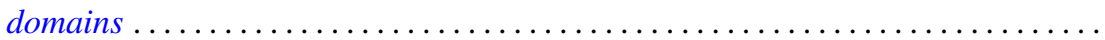

Andrzej Granas, Ronald Bernard Guenther and John Walter Lee, On a theorem of S.

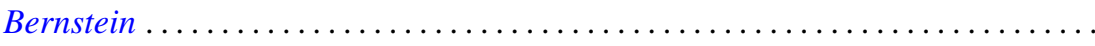

Jerry Grossman, On groups with specified lower central series quotients . .........

William H. Julian, Ray Mines, III and Fred Richman, Algebraic numbers, a constructive development . . . . . . . . . . . . . . . . . . . . . . .

Surjit Singh Khurana, A note on Radon-Nikodým theorem for finitely additive

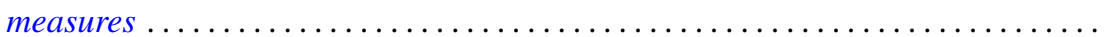

Garo K. Kiremidjian, A Nash-Moser-type implicit function theorem and nonlinear

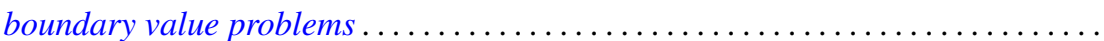

Ronald Jacob Leach, Coefficient estimates for certain multivalent functions ....

John Alan MacBain, Local and global bifurcation from normal eigenvalues. II . . 133

James A. MacDougall and Lowell G. Sweet, Three dimensional homogeneous algebras...

John Rowlay Martin, Fixed point sets of Peano continua ......

R. Daniel Mauldin, The boundedness of the Cantor-Bendixson order of some analytic sets...

Richard C. Metzler, Uniqueness of extensions of positive linear functions ..

Rodney V. Nillsen, Moment sequences obtained from restricted powers . .

Keiji Nishioka, Transcendental constants over the coefficient fields in differential elliptic function fields...

Gabriel Michael Miller Obi, An algebraic closed graph theorem

Richard Cranston Randell, Quotients of complete intersections by $\mathbf{C}^{*}$ actions . . 221

Bruce Reznick, Banach spaces which satisfy linear identities . .

Bennett Setzer, Elliptic curves over complex quadratic fields...

Arne Stray, A scheme for approximating bounded analytic functions on certain subsets of the unit disc.

Nicholas Th. Varopoulos, A remark on functions of bounded mean oscillation and bounded harmonic functions. Addendum to: "BMO functions and the

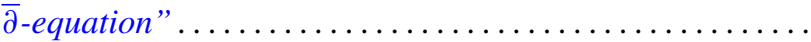

Charles Irvin Vinsonhaler, Torsion free abelian groups quasi-projective over their

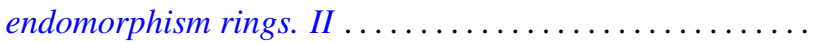

Thomas R. Wolf, Characters of $p^{\prime}$-degree in solvable groups ... 